\title{
Discovery of variable X-ray absorption in the cTTS AA Tauri
}

\author{
J. H. M. M. Schmitt and J. Robrade
}

Hamburger Sternwarte, Gojenbergsweg 112, 21029 Hamburg, Germany

e-mail: jschmitt@hs.uni-hamburg.de

Received 3 November 2006 / Accepted 6 December 2006

\section{ABSTRACT}

\begin{abstract}
We present XMM-Newton X-ray and UV observations of the classical T Tauri star AA Tau covering almost two rotational periods where $P_{\text {rot }} \sim 8.5$ days. Clear, but uncorrelated variability is found at both wavebands. The variability observed at $\sim 2100 \AA$ follows the previously known optical period. Spectral analysis of the X-ray data results in significant variability in the X-ray absorption such that the times of maximal X-ray absorption and UV extinction coincide. Placing the coronal emission in regions at low up to moderate magnetic latitudes and attribution of the variable X-ray absorption to accretion curtains and/or the disk warp provides a consistent physical picture. However, the derived X-ray absorption and optical extinction at times of maximal optical/UV brightness, i.e. outside occultation, are difficult to reconcile, requiring additional absorption in a disk wind or a peculiar dust grain distribution.
\end{abstract}

Key words. X-rays: stars - stars: pre-main sequence - stars: coronae - stars: activity

\section{Introduction}

T Tauri stars (TTS) are very young low-mass stars evolving towards the main sequence in the Hertzsprung-Russell diagram to eventually become solar-like main sequence stars. A detailed account of the pre-main sequence stellar evolution of low-mass stars is given by Feigelson \& Montmerle (1999). The subclass of "classical" TTS (cTTS) shows signs of ongoing accretion from a circumstellar disk as evidenced by a strong infrared excess and a large $\mathrm{H} \alpha$ equivalent width $(E W>10 \AA)$. The currently accepted cTTS paradigm envisages a magnetic field anchored in the star to disrupt the accretion disk in the vicinity of the corotation radius. There, material is loaded onto the magnetic field lines and falls along the field lines onto the star.

The cTTS AA Tau shows almost all the features required by this paradigm. Extensive studies by Bouvier et al. (1999) and Bouvier (2003) show AA Tau to be a rather typical cTTS with spectral type $\mathrm{K} 7$, a mass of $\sim 0.8 M_{\odot}$, and radius $\sim 1.85 R_{\odot}$. Its $\mathrm{H}_{\alpha}$ emission line $E W$ of $\sim 100 \AA$ and IR excess indicate the presence of a disk, which is seen at a rather high inclination of $i \approx 75^{\circ}$. This value and that of $v \sin (i)\left(\approx 11 \mathrm{~km} \mathrm{~s}^{-1}\right)$ lead to a rotation period of $\sim 8.5$ days. There are no indications from spectroscopic or interferometric studies that AA Tau contains a stellar companion, yet photometry of the system shows a recurrent period in the range of 8.2 to 8.5 days. Attributing the $2 \mathrm{~km} \mathrm{~s}^{-1}$ radial velocity variations (Bouvier et al. 2003) to a putative lower mass companion results in a mass of $\approx 20 M_{\text {Jupiter }}$, i.e., an object far to small to produce any significant X-ray emission. The colors of AA Tau at maximum brightness suggest only moderate extinction values, yet quite large amplitude variations of the central star are observed $(\Delta V \sim 1.4)$, that are found to be almost achromatic. The phase-folded light curves of AA Tau do change, but the times of minimum brightness for the system are restricted to $\approx 20 \%$ of the recurrence period of $\sim 8.5$ days. Bouvier et al. (1999) interpret these observational findings in terms of a "disk warp", i.e., a disk extension of a rather limited vertical, but a substantial azimuthal, extent at a distance of $\approx 8.8 R_{*}$ from the stellar surface. Because of AA Tau's peculiar viewing geometry, this warp leads to a partial occultation of AA Tau's photosphere, thus causing the observed almost achromatic brightness variations. Polarization measurements (Ménard et al. 2003) as well as hydrodynamical simulations by O'Sullivan et al. (2005) fully support this interpretation.

TTS are known to be emitters of copious X-ray emission, as shown by numerous studies with the Einstein and ROSAT observatories. The origin of the observed X-ray activity is currently the subject of considerable debate. TTS are fast rotators and have deep outer convective envelopes. This includes both types of TTS, the cTTS class of accretors and the socalled "weak-line" TTS (wTTS) with lower $\mathrm{H}_{\alpha}$-emission $(E W<$ $10 \AA$ ) and with little or no IR excess and hence thought to be disk-less. Since all such "cool stars" are surrounded by hot coronae according to a central result of stellar X-ray astronomy (Schmitt \& Liefke 2004), some scaled-up version of solar activity is expected also for those stars. The presence of accretion and winds, however, opens up additional possibilities for generating X-rays in cTTS. X-ray jets have been observed in the Herbig-Haro objects HH 2 (Pravdo et al. 2001) and HH 154 (Favata et al. 2006) or postulated in the cTTS DG Tau (Güdel et al. 2005). Reconnection between magnetic fields anchored in the disk and those on the star has been invoked to explain large flares (Favata et al. 2005). Finally, the observed anomalous line ratios between forbidden and intercombination lines in the O VII and Ne IX triplets for the cTTS TW Hya (Kastner et al. 2002; Stelzer \& Schmitt 2004), BP Tau (Schmitt et al. 2005) and V4046 Sgr (Günther et al. 2006) have been interpreted as evidence of X-ray emission from strong shocks at the bottom of a magnetic accretion funnels.

X-ray emission from AA Tau was first reported by Walter \& Kuhi (1981); it was also detected as a weak X-ray source in the ROSAT all-sky survey (RASS; Neuhäuser et al. 1995). Walter \& Kuhi (1981) detected AA Tau with an Einstein IPC rate of 0.03 IPC cts/s, but one year later AA Tau could not be detected with an upper limit of $<0.004$ IPC cts/s. The RASS rate of 0.14 PSPC cts/s is actually consistent with this upper limit, but not with an IPC rate of $0.03 \mathrm{cts} / \mathrm{s}$, indicating substantial 
variability in AA Tau's X-ray flux. In this letter we present new $\mathrm{X}$-ray observations of AA Tau carried out by XMM-Newton in February 2003 covering nearly two of AA Tau's rotational periods of $\sim 8.5$ days.

\section{Observations and data reduction}

AA Tau was observed by XMM-Newton ${ }^{1}$ on eight occasions in February 2003 for about $20 \mathrm{ks}$ each, separated by approximately two days (Table 1). The medium filter was used for all EPIC instruments, which were operated in full frame mode. The $X M M$ Newton optical monitor (OM) was operated with the UVW2 filter, which is sensitive in the waveband between 1800-2500 with an effective wavelength of $2120 \AA$. The OM exposures typically lasted between 800-1340 s, 2 or 3 exposures of the central field containing AA Tau were taken during each X-ray observation.

The data were analyzed with the XMM-Newton Science Analysis System (SAS) version 7.02. Unfortunately, during all observations, periods with large proton fluxes leading to enhanced background were encountered. AA Tau was detected in all eight observation intervals both in the EPIC cameras and in the OM data. We proceeded to produce X-ray light curves and medium-resolution X-ray spectra for all individual observations. EPIC light curves were produced by subtracting background as determined from a nearby source-free region. To generate the $\mathrm{X}$-ray spectra, we applied standard filter criteria rejecting all data with high background rates in order to avoid contamination of the derived X-ray spectra.

The light curves of AA Tau measured both in the OM UVW2 filter and the EPIC pn instrument are shown in Fig. 1. In order to convert from the actually measured count rates to magnitudes, we used the conversion factors given in XMM-Newton Science Analysis System Users' Guide. For comparison we also show the OM light curves for two check stars, LDN 15296 (spectral type: A7) and LDN 152940 (G8; cf., Feigelson \& Kriss 1983), which were produced in precisely the same fashion as that for AA Tau. An inspection of Fig. 1 shows obvious variability for AA Tau's OM light curve, while the OM light curves of the two check stars are constant within the measurement errors. While a period determination with eight, rather sparsely sampled data points is not really meaningful, it is also clear that the observed OM variability in AA Tau is consistent with a presumed $8.5 \mathrm{~d}$ period and thus consistent with the previously determined optical period of AA Tau. The X-ray lightcurve of AA Tau (bottom panel of Fig. 1) exhibits large variations (Feb. 16 and 26), and specifically the X-ray flux of AA Tau on Feb. 16, 2003 was enhanced by approximately a factor of 20-50. Further, low-level variations are present in the X-ray light curve, which could be related to rotational modulation; however, no clear periodicity is found in the existing X-ray data. We also inspected all individual X-ray observations for short-term X-ray variability. The X-ray flux of AA Tau decreased somewhat in the first few kiloseconds of the Feb. 16 observation and then stayed essentially constant for the rest of the observation. Therefore the light curve observed on Feb. 16 cannot be interpreted as a portion of a possible long-duration event, so we conclude that AA Tau was in a state of enhanced activity on that date.

1 The satellite and its instruments have been described in a Special Issue of Astronomy \& Astrophysics Vol. 365 (Jan. 2001).

${ }^{2}$ See http://xmm.esa.int/external/xmm-user-support/ documentation/sas-usg/USG/USG.html

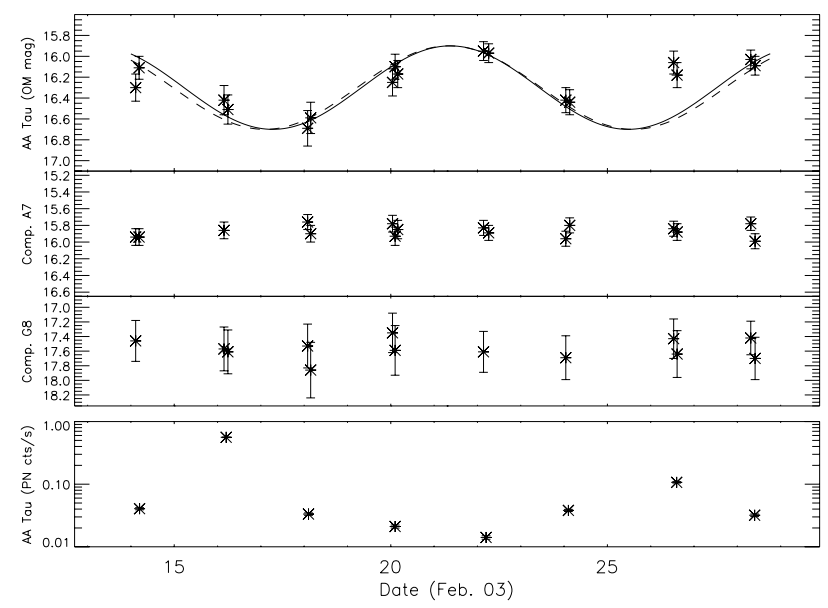

Fig. 1. OM UVW2 lightcurve of AA Tau in the OM magnitude system (upper panel); sine-curves with $p=8.2 \mathrm{~d}$ and $8.5 \mathrm{~d}$ are overplotted. Comparison light curves for the stars LDN 15296 and LDN 152940 are shown in the middle panels. The EPIC pn light curve (1.0-7.5 keV) for AA Tau is plotted in the bottom panel; all errors are $1 \sigma$.

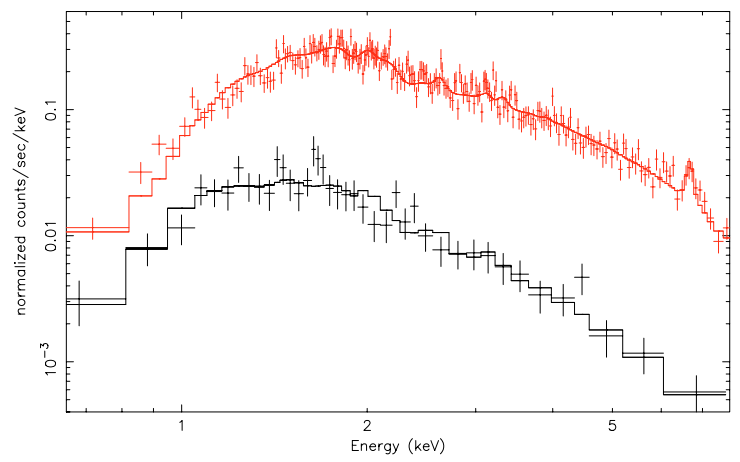

Fig. 2. EPIC pn X-ray spectra of AA Tau (crosses) with $1 \sigma$ errors including spectral fit (solid line histogram) and assuming an isothermal plasma (vapec) for the observations on Feb. 14 (black) and on Feb. 16 (red); see Table 1 for model parameters. Note both the change in flux level and photoelectric absorption.

For each observation interval, we extracted the X-ray spectra from AA Tau in the MOS1, MOS2, and pn detectors and constructed the appropriate response files. The spectral analysis of the X-ray data was carried out with XSPEC version 11.3. To increase the signal, we fitted the data from all three detectors simultaneously by first deriving an abundance pattern identical for all observations from an absorbed thermal emission model with variable abundances (vapec). We first derived abundances from a global fit of all merged observations and found a consistent solution with the (relative-to-solar) abundance of the low FIP elements (Fe, $\mathrm{Mg}$ ) set to 0.2 , the medium FIP elements (C, N, O, S, $\mathrm{Si}$ ) to 0.5 and the high FIP elements $(\mathrm{Ne}, \mathrm{Ar})$ to 1.5. Keeping the thus derived abundances fixed, we proceeded to fit the individual $\mathrm{X}$-ray spectra with a temperature, an equivalent hydrogen column density, and an overall normalization. We found that such one temperature models adequately describe all data and, in addition, ensure a consistent analysis; note that we did not allow for any constant offsets between the different detectors. The X-ray spectrum of AA Tau (cf., Fig. 2) is characterized by very large photoelectric absorption. We used $\chi^{2}$ statistics and binned the data to at least 15 events in each spectral bin. AA Tau's X-ray signal is highly variable and at times quite low; therefore this procedure leads to spectral bins with bin widths in excess of the spectral resolution, especially at the low-energy edge of the detectors. To provide robust fits, we restricted the energy range to 
Table 1. Observations and fit results for AA Tau from EPIC data, exposure times (ks) denote both the total and the filtered exposures for MOS and pn. $L_{\mathrm{X}}$ (obs./emitted) are given in the $0.2-10.0 \mathrm{keV}$ band. Units are $N_{\mathrm{H}}$ in $10^{22} \mathrm{~cm}^{-2}, k T$ in $\mathrm{keV}, E M$ in $10^{52} \mathrm{~cm}^{-3}$, and $L_{\mathrm{X}}$ in $10^{30} \mathrm{erg} \mathrm{s}^{-1}$.

\begin{tabular}{llrrrrr}
\hline \hline Date & Exposure & $N_{\mathrm{H}}$ & $k T$ & $E M$ & $L_{\mathrm{X}}$ & $\chi^{2}$ (d.o.f.) \\
\hline $2 / 14$ & $17 / 16 / 12$ & $0.99_{-0.12}^{+0.13}$ & $2.38_{-0.15}^{+0.41}$ & $9.0_{-1.2}^{+1.3}$ & $0.48 / 1.11$ & $68.7(73)$ \\
$2 / 16$ & $17 / 16 / 12$ & $1.70_{-0.05}^{+0.05}$ & $3.86_{-0.18}^{+0.19}$ & $141.4_{-5.3}^{+5.4}$ & $9.64 / 20.8$ & $835(801)$ \\
$2 / 18$ & $17 / 13 / 9$ & $1.41_{-0.30}^{+0.27}$ & $2.07_{-0.63}^{+0.69}$ & $10.2_{-2.4}^{+2.4}$ & $0.42 / 1.20$ & $44.0(42)$ \\
$2 / 20$ & $17 / 16 / 11$ & $1.29_{-0.32}^{+0.29}$ & $2.08_{-0.49}^{+0.94}$ & $6.3_{-1.7}^{+2.0}$ & $0.28 / 0.73$ & $42.1(36)$ \\
$2 / 22$ & $20 / 17 / 9$ & $0.90_{-0.43}^{+0.46}$ & $1.83_{-0.44}^{+0.82}$ & $3.5_{-1.1}^{+1.5}$ & $0.15 / 0.40$ & $22.6(21)$ \\
$2 / 24$ & $19 / 16 / 10$ & $1.73_{-0.20}^{+0.30}$ & $3.42_{-0.64}^{+0.51}$ & $9.0_{-1.6}^{+2.1}$ & $0.55 / 1.27$ & $50.2(58)$ \\
$2 / 26$ & $17 / 14 / 7$ & $1.16_{-0.10}^{+0.11}$ & $3.89_{-0.51}^{+0.66}$ & $21.6_{-2.0}^{+2.1}$ & $1.64 / 3.18$ & $139(134)$ \\
$2 / 28$ & $17 / 13 / 8$ & $1.33_{-0.36}^{+0.20}$ & $2.43_{-0.49}^{+0.81}$ & $9.3_{-2.2}^{+2.7}$ & $0.48 / 1.16$ & $30.3(41)$ \\
\hline
\end{tabular}

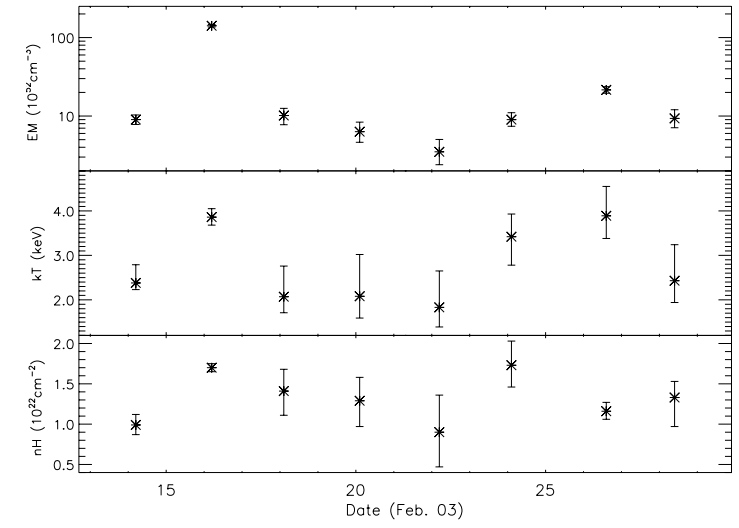

Fig. 3. Time-dependent emission measure (upper panel), temperature (middle panel) and absorption column density (bottom panel) as derived from the XMM-Newton EPIC spectra; all errors are $90 \%$.

1.0-7.0 keV for data with fewer than 50 spectral bins in the EPIC detectors; otherwise, the full energy range between $0.3-10.0 \mathrm{keV}$ is used. The derived fit parameters and their errors (90\% confidence) are given in Table 1.

As an example of the quality of the data and of our model fits we show the pn data and the best fit models for the first two observations in Fig. 2, the upper spectrum is the one recorded with the largest flux, clearly showing the $6.7 \mathrm{keV}$ iron "line". Figure 2 demonstrates, first, that our simple one-temperature model with photoelectric absorption does indeed provide a good description of the data, and, second that the different slope of the recorded spectra between 1 and $2 \mathrm{keV}$ is a direct indicator of the variable absorption between the two data sets. As is apparent from Table 1 and Fig. 3, large flux and absorption variations do occur between these two data sets. The simplest spectral model to adequately describe the observed X-ray data is a 1-T thermal model, which, however, need not necessarily be the physically correct model. For example, an accretion funnel shock with temperatures of, say, $2 \mathrm{MK}$, will produce soft X-ray emission around $0.5 \mathrm{keV}$, which is not accounted for by our onecomponent model. If additional soft X-ray emission is present, the required absorption column density increases to ensure compatibility with the observed X-ray flux.

\section{Discussion and conclusions}

Are the XMM-Newton observations compatible with the warped disk scenario and what is the origin of AA Tau's X-ray emission? To address these issues it is useful to visualize the model geometry proposed by Bouvier et al. (1999), including the

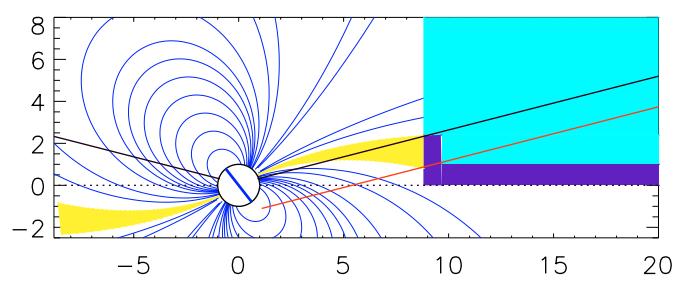

Fig. 4. Sketch of the model geometry of AA Tau and its disk. All units are in stellar radii. The $x$-axis represents the plane of the disk and some field lines (blue) of a dipole inclined by $52^{\circ}$ and the magnetic equator are also shown. The system is symmetric w.r.t. the disk plane, except for the warp. The observer is viewing into the system at an angle of $15^{\circ}$ at the phase of minimal/maximal optical/UV brightness (solid black lines); the red line is the line of sight towards a possible X-ray corona in the magnetic equatorial regions. The yellow region represents the accretion curtain, the dark blue regions the disk warp and an outer thinner disk, and the turquoise region the assumed wind zone.

presumed field lines of a magnetic dipole (see Fig. 4). At the time of maximum obscuration, an outside observer's line of sight is inclined by $15^{\circ}$ with respect to the disk and just grazes the disk warp. Obviously one expects to see an increase in absorption at that phase, while X-ray and optical flux need not be correlated. Given the high X-ray temperatures (see Table 1) and the detection of the Fe K "line" at $6.7 \mathrm{keV}$, it is clear that such a high plasma temperature cannot be produced by a simple conversion of kinetic into thermal energy in a strong shock, such that the observed X-ray emission must be produced in some other way.

Figure 1 demonstrates that the OM UVW2 light curve of AA Tau is consistent with the previously determined period of 8.2-8.5 days. The blue $U V W 2$ filter is particularly sensitive to the flux emitted from the accretion spot regions near the surface of AA Tau. Interpreting the XMM-Newton data in the framework of the warped accretion disk model, we identify the times of minimal UV brightness with those phases when the observer's line of sight grazes the maximal disk warp with about half of the accretion region being occulted. Clearly, the X-ray flux does not follow the UV count rate, and the two data sets are essentially uncorrelated, as demonstrated by Fig. 5 (lower panel); a formal correlation analysis yields correlation coefficients $\rho=0.04$. This suggests that the production of the X-rays and UV flux is independent. Conversely, X-ray absorption and OM count rate are correlated (Fig. 5, upper panel) with a correlation coefficients $\rho=-0.67$. It is thus natural to also attribute the observed "excess" absorption to the disk warp. From Table 1 we find the difference between maximal and minimal cold absorption to be $\approx 8 \times 10^{21} \mathrm{~cm}^{-2}$ of equivalent hydrogen column, which would then be the required column density along the line of sight through the disk warp.

To determine whether the association of X-ray absorption and disk warp is physically consistent, let us first consider the absorption derived at phases when AA Tau is not occulted by its own disk. A comparison of the absorption column derived for AA Tau with those found for other cTTS (Robrade \& Schmitt 2006) at the same distance (BP Tau: $N_{\mathrm{H}, \mathrm{BP} \text { Tau }}=1.5 \times 10^{21} \mathrm{~cm}^{-2}$; SU Aur: $N_{\mathrm{H}, \mathrm{SU} \text { Aur }}=3.1 \times$ $10^{21} \mathrm{~cm}^{-2}$ ) leads us to conclude that the column towards AA Tau is exceptionally large compared to other cTTS. The use of equivalent hydrogen absorption columns is standard practice in $\mathrm{X}$-ray astronomy. In actual fact, the absorption at energies below $\sim 1.5 \mathrm{keV}$ is not due to hydrogen, but instead due to (mostly) oxygen and iron. Similarly, the extinction in the optical is due to dust grains, which are also predominantly composed of elements heavier than hydrogen and helium. 


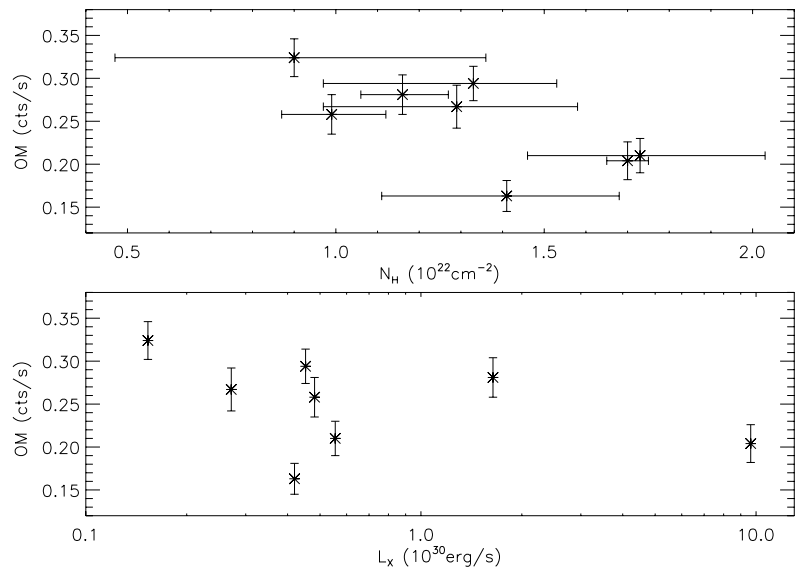

Fig. 5. Scatter plot between OM UVW2 count rate and X-ray absorption (upper panel) and X-ray luminosity (lower panel) respectively.

For absorption in the interstellar medium (ISM), one finds an empirically derived "canonical" conversion between total hydrogen column density and color excess of $N_{\mathrm{H}}=5 \times$ $10^{21} E_{B-V}$ (Bohlin et al. 1978) and $E_{B-V}=A_{V} / R_{V}$ with $R_{V} \sim 3.1$ (Cardelli et al. 1989) for that between color excess and extinction. Bouvier et al. (1999) and Hartigan et al. (1995) quote extinctions of $A_{V}=0.76$ and $A_{V}=1.3$, respectively, for AA Tau. Using the above conversions we find an equivalent column density of $0.12-0.21 \times 10^{22} \mathrm{~cm}^{-2}$, which is far lower than the absorption columns derived from the X-ray data (cf., Table 1). Admittedly, the parameter $R_{V}$ differs for different lines of sight; however, to reconcile our derived $N_{\mathrm{H}}$ columns at maximal optical light with an extinction of $A_{V}=0.76$ requires $R_{V} \sim 0.4$, a rather unusual value. Similar problems were encountered by (Güdel et al. 2005) in their analysis of the X-ray spectrum of the cTTS DG Tau.

To reconcile the observed extinction and X-ray absorption, one needs X-ray absorption in an optically almost transparent medium. From Fig. 4 it appears that an obvious candidate would be a wind thought to emanate from the disk. Assuming ad hoc that the wind emission occurs between $10<R_{*}<20$ leads to wind densities on the order $n_{\mathrm{W}} \sim 10^{10} \mathrm{~cm}^{-3}$ to produce the required columns; also, the wind must not contain dust and has to be cool enough to avoid producing any soft X-ray emission. With an ad hoc outflow speed of $100 \mathrm{~km} \mathrm{~s}^{-1}$, the mass-loss rate of such a disk wind would be $\sim 4 \times 10^{-8} \dot{M}_{\odot} /$ yr, i.e., on the order of the presumed accretion rate.

Let us now turn to the "excess" absorption of $\approx 8 \times 10^{21} \mathrm{~cm}^{-2}$ observed at the time of optical/UV minimum. The column density $N_{\text {acc }}$ of the accretion flow along the length $L_{\text {acc }}$ between disk warp and stellar surface can be estimated from $N_{\text {acc }}=\frac{\dot{M} L_{\text {acc }}}{f R} \frac{1}{4 \pi m_{\mathrm{H}} \sqrt{2 G M R}}$, where $\dot{M}$ denotes the mass accretion rate and $f$ the accretion filling factor. With $\log \dot{M} \sim-6.9 M_{\odot} / \mathrm{yr}$ Hartigan et al. (1995), and assuming a typical filling factor $f=$ 0.01 (Calvet \& Gullbring 1998) one obtains $N_{\text {acc }}$ in the range $10^{25}-10^{26} \mathrm{~cm}^{-2}$. Therefore, one expects optically thick conditions also under inclined lines of sights and it should be straightforward to produce the required absorption columns of $10^{22} \mathrm{~cm}^{-2}$. Thus lines of sight through the accretion curtain are expected to produce the required absorption column at X-ray wavelengths, and being ionized, the accretion streams should be optically transparent. The X-ray emitting material would have to be located in a small region at medium magnetic latitudes; see Fig. 4. Alternatively, one might envisage the X-ray emitting material located "underneath" the warped disk at lower magnetic latitudes. Converting the observed "excess" absorption with the "standard" conversion yields an $A_{V} \sim 5$, sufficient for explaining the observed optical magnitude dips. However, the usual ISM grain-size distributions would lead to a color dependence of the light curve, which is observationally constrained. Grain-size distributions and extinction have been studied by (Weingartner \& Draine 1981), who showed that the distributions differ in different environments and that a substantial number of very small grains must exist to account for the observed short-wavelength infrared emission. The grain size distribution in AA Tau's disk is unknown, and it remains unclear if grain size distributions consistent with the required X-ray absorption and the color-independent optical/IR light curve can be found.

In summary, the XMM-Newton data support - by and large the warped disk scenario; however, it is clear from our spectral fits to the EPIC data that a "partial coverage" model as proposed to the explain the optical/IR light curves of AA Tau cannot hold for a hypothesized corona around AA Tau, since the unocculted part of the corona would contribute to the X-ray flux at soft energies. Rather, the corona has to be located either at medium magnetic latitudes if the "excess" absorption comes from the accretion curtain or at low magnetic latitudes if it is due to the disk. To be consistent with the observed optical extinction, the large X-ray absorption observed outside eclipse requires an optically more or less transparent medium, possibly in a wind emanating from AA Tau's disk or from peculiar grains surrounding AA Tau. Any successful model for explaining the X-ray emission of AA Tau must thus account, first, for the exceptionally large absorption of the X-ray emission and, second, for the observed "reddening" of the X-ray spectrum, given the fact that the extinction towards AA Tau in its unocculted state is quite small $\left(A_{V}=0.76 \ldots 1.3\right)$, that the optical light curve modulations are high $(\Delta V \sim 1.5 \mathrm{mag})$ and essentially grey, and that those observed at $\sim 2100 \AA$ are low $(\Delta U V W 2 \sim 0.7 \mathrm{mag})$ compared to the optical light variations.

Acknowledgements. This work is based on observations obtained with $X M M$-Newton, an ESA science mission with instruments and contributions directly funded by ESA Member States and the USA (NASA). J.R. acknowledges support from the DLR under grant 50OR0105.

\section{References}

Bohlin, R. C., Savage, B. D., \& Drake, J. F. 1978, ApJ, 224, 132 Bouvier, J., Grankin, K. N., Alencar, S. H. P., et al. 1999, A\&A, 349, 619

Bouvier, J., Chelli, A., Allain, S., et al. 2003, A\&A, 409, 169

Calvet, N., \& Gullbring, E. 1998, ApJ, 509, 802

Cardelli, J. A., Clayton, G. C., \& Mathis, J. S. 1989, ApJ, 345, 245

Favata, F., Flaccomio, E., Reale, et al. 2005, ApJS, 160, 469

Favata, F., Bonito, R., Micela, G., et al. 2006, A\&A, 450, L17

Feigelson, E. D., \& Kriss, G. 1983, AJ, 88, 431

Feigelson, E. D., \& Montmerle, T. 1999, ARA\&A, 37, 363

Güdel, M., Skinner, S. L., Briggs, K. R., et al. 2005, ApJ, 626, L53

Günther, H. M., Liefke, C., Schmitt, et al. 2006, A\&A, 459, L29

Hartigan, P., Edwards, S., \& Ghandour, L. 1995, ApJ, 452, 736

Kastner, J. H., Huenemoerder, D. P., Schulz, N. S., et al., 2002, ApJ, 567, 434

Ménard, F., Bouvier, J., Dougados, C., et al. 2003, A\&A, 409, 163

Neuhäuser, R., Sterzik, M. F., Schmitt, J. H. M. M., et al. 1995, A\&A, 297, 391

O’Sullivan, M., Truss, M., Walker, C., et al. 2005, MNRAS, 358, 632

Pravdo, S. H., Feigelson, E. D., Garmire, et al. 2001, Nature, 413, 708

Robrade, J., \& Schmitt, J. H. M. M. 2006, A\&A, 449, 737

Schmitt, J. H. M. M., \& Liefke, C. 2004, A\&A, 417, 651

Schmitt, J. H. M. M., Robrade, J., Ness, J.-U., et al. 2005, A\&A, 432, L35

Stelzer, B., \& Schmitt, J. H. M. M. 2004, A\&A, 418, 687

Walter, F. M., \& Kuhi, L. V. 1981, ApJ, 284, 194

Weingartner, J. C., \& Draine, B. T. 2001, ApJ, 548, 296 